\title{
Namenslisten bei Entlassungen - Ambivalentes Mittel für Betriebsräte
}

Will ein Arbeitgeber wegen einer Betriebsänderung mehreren Arbeitnehmern kündigen, kann er die Auswahl gemeinsam mit dem Betriebsrat treffen. Werden die Ausgewählten namentlich aufgelistet, wird gemäß $₫ 1$ Abs. $5 \mathrm{KSchG}$ der Kündigungsschutz für die Betroffenen eingeschränkt. Diese im Kündigungsschutzgesetz vorgesehene Regelung ist rechtspolitisch umstritten. Die Argumente kreisen auch um die Fragen, wie weit durch Namenslisten der Kündigungsschutz beschnitten wird und ob sie dem Betriebsrat eine sinnvolle Mitwirkungsmöglichkeit bei der Auswahl der Arbeitnehmer eröffnen. Fraglich ist auch, ob die Handlungsmacht der Betriebsräte ausreicht, um die Rechtmäßigkeit der Kündigung sicherzustellen. Empirische Befunde liefert die WSI-Betriebsrätebefragung 2007.

\section{Einleitung}

Um die 1996 erstmals eingeführte Regelung zu Namenslisten bei Kündigungen ( $\$ 1$ Abs. $5 \mathrm{KSchG)}$ entbrannten immer wieder rechtspolitische Diskussionen. 1998 war sie zunächst wieder aufgehoben worden, wurde aber 2004 wortgleich erneut eingeführt. 1996 wie auch 2004 nannte der Gesetzgeber mehr „Transparenz und Rechtssicherheit" als Ziel (Deutscher Bundestag 1996, S. 8; ders. 2003, S. 10). Das angeblich komplizierte und unübersichtliche Kündigungsrecht führe zu hohen Gerichtskosten, damit zu hohen Beschäftigungskosten und letztlich zu einer psychologischen Barriere für Neueinstellungen (Deutscher Bundestag 2003, S. 8). Mehr Rechtssicherheit sollte zusätzliche Beschäftigung bewirken. Diese behaupteten Wirkungen des Kündigungsschutzes sind allerdings durch zahlreiche empirische Studien widerlegt (Pfarr et al. 2005; OECD 1999; 2006), die größtenteils schon 2004 bekannt waren, aber vom Gesetzgeber ignoriert wurden. Der aktuelle Entwurf für ein Arbeitsvertragsgesetz (Henssler/Preis 2007) sieht ebenfalls in $\$ 117$ Abs. 4 eine entsprechende Regelung vor.

\section{Voraussetzungen und Folgen von Namenslisten}

Eine Namensliste schränkt die Möglichkeiten zur Überprüfung der sozialen Rechtfertigung einer Kündigung im Kündi- gungsschutzprozess ein. Der sonst notwendige betriebliche Grund für die Kündigung wird vom Gesetzgeber vermutet, sodass die soziale Auswahl nur auf grobe Fehlerhaftigkeit überprüfbar ist.

\subsection{SITUATION OHNE NAMENSLISTE}

Ohne eine gültige Namensliste muss der Arbeitgeber zunächst die dringenden betrieblichen Erfordernisse für die Kündigung im Prozess darlegen und beweisen. Schon in einem normalen Kündigungsschutzprozess ohne Namensliste ist die Überprüfung des betrieblichen Grundes durch die Arbeitsgerichte nicht sehr weitreichend. Die unternehmerische Entscheidung wird von den Gerichten akzeptiert, es sei denn, sie ist offensichtlich unsachlich, unvernünftig oder willkürlich; die Zweckmäßigkeit wird nicht überprüft. Als Grund für den Arbeitsplatzwegfall kann auf außerbetriebliche Entwicklungen oder auf eine Entscheidung der Unternehmensleitung verwiesen werden. Allerdings werden durch Tatsachen belegte Ausführungen zu der Entscheidung verlangt, aufgrund derer der Arbeitsplatz des Arbeitnehmers entfallen ist. Der Arbeitnehmer kann entgegenhalten, es gebe im Unternehmen die Möglichkeit einer anderweitigen Beschäftigung. Dann muss der Arbeitgeber beweisen, dass eine solche Beschäftigungsmöglichkeit nicht besteht.

Zweite Voraussetzung für die soziale Rechtfertigung betriebsbedingter Kündigungen ist eine Auswahl der Arbeitnehmer nach den sozialen Kriterien des Kündigungsschutzgesetzes (Dauer der Betriebszugehörigkeit, Lebensalter, Unterhaltspflichten und Schwerbehinderung). Der Arbeitgeber kann ein Punkteschema ver- wenden, das jedem Arbeitnehmer für jedes der vier Kriterien eine bestimmte Zahl von Punkten zuweist, die addiert werden. Er muss eine abschließende Einzelfallbetrachtung vornehmen, damit besondere Belastungen, denen nach dem Punkteschema nicht Rechnung getragen werden kann, auch berücksichtigt werden.

Vor der Sozialauswahl müssen die Arbeitnehmer bestimmt werden, die für die Kündigungen überhaupt infrage kommen. Das sind alle Arbeitnehmer, die mit jenen vergleichbar sind, deren Arbeitsplätze wegfallen. Vergleichbar sind Arbeitnehmer, die entsprechende Fähigkeiten haben und denen entsprechende Tätigkeiten zugewiesen werden können. Das Gesetz lässt zwei Ausnahmen zu: Arbeitnehmer, die der Arbeitgeber behalten möchte, können nach $\$ 1$ Abs. 3 S. 2 KSchG von vornherein aus der Sozialauswahl ausgeklammert werden, „wenn deren Weiterbeschäftigung ... im berechtigten betrieblichen Interesse liegt". Die Regelung zielt zum einen auf „Leistungsträger“, die z. B. wichtige Kunden-

Stefanie Kremer, Dr., war Wissenschaftlerin im WSI in der Hans-Böckler-Stiftung, Projekt Regulierung des Arbeitsmarktes (REGAM) bis April 2008.

e-mail: stefkremer@gmx.de Nadine Zeibig ist Wissenschaftlerin im WSI in der Hans-Böckler-Stiftung, Projekt Regulierung des Arbeitsmarktes (REGAM). e-mail: nadine-zeibig@boeckler.de

Die Autorinnen danken Wolfram Brehmer, WSI, für zahlreiche Berechnungen zu teilweise sehr speziellen Fragestellungen. 
kontakte betreuen, besondere Spezialisierungen oder einen betriebsspezifischen Erfahrungsschatz aufgebaut haben. Um eine ausgewogene Altersstruktur im Betrieb zu bewahren, erlaubt die Regelung daneben, jüngere Arbeitnehmer aus der Sozialauswahl auszuklammern. Beides sollen aber Ausnahmen bleiben.

\subsection{SITUATION MIT NAMENSLISTE}

Gibt es eine gültige Namensliste, wird das Vorliegen eines dringenden betrieblichen Grundes vom Gericht vermutet (widerlegliche gesetzliche Vermutung). Will der Arbeitnehmer die Kündigung vor Gericht erfolgreich angreifen, muss er beweisen, dass seine Kündigung nicht durch dringende betriebliche Gründe gerechtfertigt ist. Hierfür reicht es nicht, wenn er Tatsachen vorträgt, die an dem Vorhandensein eines betrieblichen Grundes nur Zweifel aufkommen lassen.

Regeln zur Verteilung der Beweispflicht einschließlich gesetzlicher Vermutungen sind immer nötig, da eine gerichtliche Entscheidung auch gefällt werden muss, wenn ein Sachverhalt nicht gänzlich aufgeklärt werden kann - eine verbreitete Situation. Ist das der Fall und gilt keine besondere Beweispflichtregelung, entscheidet das Gericht so, als ob die entscheidungsrelevante Tatsache nicht vorliegt. Denn jede Partei muss grundsätzlich darlegen und beweisen, dass der Tatbestand der ihr günstigen Gesetzesbestimmung erfüllt ist. Bei der gesetzlichen Vermutung macht der Gesetzgeber eine Ausnahme von dieser Grundregel und legt fest, dass der Richter bei fehlender Aufklärbarkeit einer entscheidungsrelevanten Tatsache so entscheidet, als ob die Tatsache, für die die Vermutung gilt, vorliegt. Aus Sicht der am Rechtsstreit beteiligten Parteien legen Regeln zur Verteilung der Beweispflicht fest, wer das Prozessrisiko trägt. $\$ 1$ Abs. 5 KSchG verlagert das Prozessrisiko auf den Arbeitnehmer und führt somit zu einer Verkürzung seines Rechtsschutzes. Allerdings müssen grundsätzlich auch bei einer gesetzlichen Vermutung beide Parteien zur Aufklärung der entscheidungsrelevanten Tatsachen beitragen, jede zu den Tatsachen, über die die andere mangels Zugang kaum Informationen haben kann. Über die betrieblichen Gründe für die Kündigung hat der Arbeitgeber grundsätzlich mehr Informationen, die er deshalb auch zur Sachverhaltsaufklärung beitragen muss, soweit sie dem Arbeitnehmer nicht zur Verfügung stehen. $₫ 138$ Abs. 2 $\mathrm{ZPO}$, in dem dieser allgemeine Grundsatz festgelegt ist, spielt immer wieder eine Rolle in der Diskussion um Namenslisten, denn je nachdem, wie er von den Arbeitsgerichten gehandhabt wird, stellt sich die Namensliste als mehr oder weniger gravierende Einschränkung des Rechtsschutzes für den Arbeitnehmer dar. Dementsprechend unterschiedlich werden in der rechtspolitischen Diskussion die Erfolgsaussichten einer Kündigungsschutzklage mit Namensliste beurteilt.

Anders als die gesetzliche Vermutung beim betrieblichen Grund berührt die beschränkte Überprüfbarkeit der Sozialauswahl bei einer Namensliste das Verfahren der Tatsachenermittlung im Prozess nicht. Die Darlegungs- und Beweispflicht für die Sozialauswahl regelt $\$ 1$ Abs. 3 S. 3 KSchG immer schon differenziert: Der Arbeitgeber muss die Kriterien darlegen, die er für die Auswahl verwendet hat; der Arbeitnehmer muss darlegen und beweisen, dass die Sozialauswahl nicht richtig durchgeführt wurde. Hieran ändert eine $\mathrm{Na}$ mensliste nichts. Die Regelung erleichtert dem Arbeitgeber die Kündigung in vergleichbarer Weise wie eine materiell-rechtliche Änderung des Kündigungsschutzgesetzes: Die in $\$ 1$ Abs. 3 KSchG geregelten Vorgaben für die Sozialauswahl (Berücksichtigung der gesetzlichen Kriterien und Verzicht auf nicht im Gesetz genannte sachfremde Kriterien) dürfen in der $\mathrm{Na}$ mensliste bis zu einem gewissen Grad ignoriert werden. Die Sozialauswahl darf nur nicht grob fehlerhaft sein; das ist sie dann, wenn zentrale Gesichtspunkte nicht in die Bewertung einbezogen worden sind und die Bewertung jede Ausgewogenheit vermissen lässt (Deutscher Bundestag 1996, S. 9). ${ }^{1}$

Damit die Rechtsfolgen des $\$ 1$ Abs. 5 KSchG ausgelöst werden, muss eine Betriebsänderung ( $\$ 111$ BetrVG) gegeben sein und die Namensliste muss bestimmte Formerfordernisse erfüllen. Eine solche Betriebsänderung liegt vor, wenn bestimmte tatsächliche Voraussetzungen erfüllt sind; beispielhaft sind in Abs. 1 S. 3 der Vorschrift einige Fälle aufgezählt; Veräußerung von Betriebsmitteln und Kündigung von Arbeitnehmern müssen jedoch nicht gemeinsam auftreten. Werden nur Arbeitnehmer gekündigt, aber keine Betriebsmittel veräußert, muss einer Mindestanzahl von Arbeitnehmern gekündigt werden.

Ganz vereinzelt wird die Auffassung vertreten, dass auch „freiwillige Namenslisten", die außerhalb einer Betriebsänderung abgeschlossen werden, die Rechtsfolgen des $\$ 1$ Abs. 5 KSchG auslösen (Kappenhagen 2004, Thüsing/Wege 2005). Die bloße Existenz einer Namensliste reiche aus. Auch Namenslisten, die vor 1996 und in den Jahren 1999 bis 2003 abgeschlossen wurden, als $\$ 1$ Abs. 5 KSchG nicht galt, werden teilweise als „freiwillige Namenslisten“ bezeichnet. Eine Recherche in der Urteilsdatenbank Juris zum Stichwort „Namensliste" ließ aber keine Anhaltspunkte dafür erkennen, dass in diesem Zeitraum von Arbeitgeberseite versucht wurde, sich in einem Kündigungsschutzprozess auf eine solche „freiwillige“ Namensliste zu berufen.

\section{Rolle der Betriebsräte}

Es gibt zahlreiche Bedenken gegenüber der Regelung des $₫ 1$ Abs. 5 KSchG. Sie verkürzt in der Tat den Kündigungsschutz, da unabhängig vom tatsächlichen Vorliegen eines betrieblichen Grundes für die Kündigung dieser von den Arbeitsgerichten vermutet und die Sozialauswahl nur noch auf grobe Fehlerhaftigkeit überprüft wird. Das veranlasst viele zu dem Urteil, dass eine Kündigungsschutzklage durch eine $\mathrm{Na}$ mensliste praktisch aussichtslos werde (Reufels 2007, S. 48).

Die Entscheidung für den Abschluss der Namensliste und damit für eine Einschränkung des Kündigungsschutzes wird den Betriebsräten auferlegt. Ihnen ist die Beteiligung an der Erstellung der Namensliste freigestellt; ihre Beteiligung kann auch nicht durch Spruch der Einigungsstelle ersetzt werden. Der Betriebsrat, dessen Aufgabe der Arbeitnehmerschutz ist, hat es damit in der Hand, zu verhindern, dass gerichtlich kaum noch angreifbare rechts-

\footnotetext{
1 Wie die Gesetzesbegründung von 2004 bestimmt, werden die beiden Punkte zur Feststellung, welche Arbeitnehmer in die Sozialauswahl einzubeziehen sind (Prüfung der Vergleichbarkeit/Ausklammerung von Leistungsträgern), bei einer Namensliste ebenfalls nur eingeschränkt überprüft (Deutscher Bundestag 2003, S. 12). Da dies für die alte Regelung von 1996 nicht galt, brachte die Wiedereinführung 2004 damit eine weitere Einschränkung des Kündigungsschutzes, die dem Gesetz selbst nicht zu entnehmen ist, von den Arbeitsgerichten aber zu beachten ist.
} 
widrige Kündigungen möglich werden. Damit trägt der Betriebsrat eine schwere Last. Zahlreiche Anzeichen deuten darauf hin, dass seine rechtliche und tatsächliche Position ihm gar nicht erlaubt, diese Last zu schultern.

\subsection{BETRIEBSRATSBETEILIGUNG: TEILERSATZ FÜR RECHTSSCHUTZ?}

Bei Namenslisten soll der Betriebsrat die sonst durch die Arbeitsgerichte durchgeführte Rechtmäßigkeitsprüfung vorwegnehmen. Dass der Namenslistenregelung dieser Gedanke zugrunde liegt, folgt auch aus den Ausführungen des Bundesarbeitsgerichts. Das Bundesarbeitsgericht hat mehrfach betont, dass die Beteiligung des Betriebsrats an der Auswahl der Arbeitnehmer eine größere Gewähr für die Fehlerfreiheit der Kündigungen bietet und dass dies die Einschränkung des Kündigungsschutzes bei einer Namensliste rechtfertigt (BAG 2007). Ähnlich formuliert Kittner für die Sozialauswahl: Der Grund dafür, dass nur „grobe Fehlerhaftigkeit“ die Kündigung unwirksam werden lässt, ist die durch Beteiligung der Betriebsvertretung unterstellte höhere Richtigkeitsvermutung zugunsten der Auswahlentscheidung (Kittner et al. 2004, S. 1533). Sowohl der betriebliche Grund als auch die von Kittner erwähnte Auswahlentscheidung bei der Sozialauswahl sollen demzufolge nach Beteiligung des Betriebsrats mit höherer Wahrscheinlichkeit den gesetzlichen Anforderungen von $\$ 1$ Abs. 2, $3 \mathrm{KSchG}$ entsprechen als in Fällen, in denen der Betriebsrat nicht mitgewirkt hat. Dass der Betriebsrat diese gerichtliche Ersatzfunktion umfassend wahrnehmen kann, ist jedoch zweifelhaft.

\subsection{MOTIVE DES BETRIEBSRATS}

Viele Arbeitgeber stellen besondere Leistungen für die Arbeitnehmer in Aussicht, um die Betriebsräte zur Beteiligung an einer Namensliste zu bewegen. Das Motiv, solche Leistungen zu erhalten, kann bei der Entscheidung des Betriebsrats, sich an einer Namensliste zu beteiligen, das Motiv der Rechtmäßigkeitskontrolle in den Hintergrund drängen. Anders ausgedrückt: Der Betriebsrat wird erpressbar. So stellen Arbeitgeber bei den Verhandlungen zu Namenslisten häufig Erhöhungen der Sozialplanabfindungen oder Beschäftigungssicherungen für die Verbleibenden in Aussicht. Solche Angebote können den Be- triebsrat in eine Situation bringen, in der er sich möglicherweise auch mit rechtlich fragwürdigen Kündigungen einverstanden erklärt. Der Betriebsrat soll zwar eigentlich "den Arbeitnehmer" schützen, da er aber Vertreter der gesamten Belegschaft ist, schützt er in der betrieblichen Praxis meist die Mehrheit der Belegschaft (Gamillscheg 2008, S. 26f.). Die Mehrheit der Arbeitnehmer wird, wenn dem Betriebsrat eine Gegenleistung vom Arbeitgeber angeboten wird, stärker profitieren, wenn der Betriebsrat sich an der Namensliste beteiligt und so die Gekündigten in den Genuss von höheren Sozialplanabfindungen kommen oder die Verbleibenden Beschäftigungssicherungen erhalten - auch wenn dabei einigen Arbeitnehmern rechtswidrig gekündigt werden sollte. Zwar betonen Lehrbücher zum Betriebsverfassungsrecht, dass der Betriebsrat grundsätzlich auch auf den einzelnen Arbeitnehmer oder eine Minderheit Rücksicht nehmen muss und dass das Interesse auch nur einer Minderheit, den Arbeitsplatz zu behalten, vorgehe, selbst wenn das bei allen den Abbau zusätzlicher Leistungen verlangt oder sich eine Abfindung für die Gekündigten vermindert (ebd., S. 29). Dies ist aber, wie sich gerade bei der Namensliste zeigt, ein Ideal, welches sich in der Praxis nicht immer wiederfindet. Wird dem Betriebsrat eine Gegenleistung in Aussicht gestellt, die der Mehrheit von Arbeitnehmern zugute kommt, scheint es nicht gerechtfertigt, sie zugunsten von Einzelinteressen auszuschlagen. Noch deutlicher als bei Abfindungserhöhungen ist die Dominanz von Mehrheitsinteressen bei dem Angebot von Beschäftigungssicherungen für die verbleibenden Beschäftigten. Unwahrscheinlich ist auch, dass die verbleibende Belegschaft eine Missachtung gesetzlicher Kriterien oder sozialer Aspekte bei der Auswahl der zu Kündigenden missbilligt, wenn deren strikte Anwendung zu ihrer eigenen Entlassung führen könnte.

\subsection{VERBUNDENHEIT DER BETRIEBSRÄTE MIT DEM BETRIEB}

Betriebsräte sind zudem selbst dem Betrieb so stark verbunden, dass sie kaum eine ähnlich neutrale Position einnehmen können wie ein Arbeitsgericht. Deshalb ist es ihnen nicht umfassend möglich, die Rechtmäßigkeit der Kündigungen sachgerecht und unabhängig zu beurteilen. Die Verbundenheit mit dem Betrieb ist einer der Gründe, warum Betriebsräte in Verhand- lungen mit dem Arbeitgeber eine unterlegene Position einnehmen, anders als z.B. Gewerkschaften. Betriebliche Vereinbarungen entstehen, wie Höland ausführt, „typischerweise aus Bedingungen, die keine Gewähr für verfahrensgerechte und ausgewogene Aushandlung von Recht bieten können. Betriebsräte bestehen aus Arbeitnehmern, die ungeachtet rechtlicher Schutzvorkehrungen dem Unternehmen vertraglich, wirtschaftlich und sozial verbunden sind. Die Mitglieder von Betriebsräten denken, wie sollte es anders sein, in den konkreten Bedingungs- und Risikolagen „ihres" Unternehmens. Ihre berufliche Entwicklung, ihre wirtschaftliche Existenz und Organstellung als Betriebsratsmitglieder lassen sich von der Existenz des Unternehmens nicht ablösen. Aus der doppelfunktionalen Stellung als Arbeitnehmer und Interessenvertreter entsteht eine Zwitterstellung, die sich nicht für denselben Grad von Unabhängigkeit von Unternehmensinteressen eignet wie ihn die Gewerkschaft erreichen kann. (...) Der Statik betrieblichen Rechtshandelns fehlt das Strukturelement gleichverteilter Handlungsmacht zwischen Betriebsrat und Arbeitgeber; dass es empirisch gelegentlich vorhanden ist, widerspricht dieser Regel nicht" (Höland 2002, S. 49).

Die unterlegene Verhandlungsposition von Betriebsräten wird vor allem durch einen Vergleich ihrer Situation mit der von Gewerkschaften deutlich. Anders als Betriebsräte stehen Gewerkschaften den Arbeitgebern und ihren Verbänden anerkanntermaßen als gleichstarke Verhandlungspartner gegenüber. Der empirische Befund in Bezug auf die unterschiedliche Verhandlungssituation von Gewerkschaften und Betriebsräten wurde zum Beispiel von den Arbeitsgerichten nie in Zweifel gezogen; sie gehen davon aus, dass betriebliche Vereinbarungen nicht in gleichem Maße wie Tarifverträge die Gewähr bieten, dass ihr Inhalt rechtmäßig ist (ebd., S. 48). Daher achten Gerichte bei betrieblichen Vereinbarungen auch auf deren „Billigkeit“, d. h. sie prüfen, ob durch sie keine der beiden Seiten übervorteilt wurde (BAG 1987), während bei Tarifverträgen eine solche inhaltliche Kontrolle nicht stattfindet. Tarifverträge haben die Vermutung für sich, dass ihre Regelungen den Interessen beider Seiten gerecht werden, weshalb sie nur unter dem Aspekt grober Grenzüberschreitungen überprüft werden (BAG 1996). Wegen der gleichgewichtigen Macht der Tarif- 
partner kann der Gesetzgeber den „verbandlichen Akteuren der Tarifautonomie ... das Gestaltungsfeld der Arbeits- und Wirtschaftsbeziehungen getrost überlassen" (Höland 2002, S. 49). Für betriebliche Vereinbarungen gilt das nicht. Es gibt folglich wenig Anlass für die Annahme des Bundesarbeitsgerichts, dass bei Namenslisten die Kündigungen bei Betriebsratsbeteiligung mit größerer Wahrscheinlichkeit rechtmäßig sind, mit der die Einschränkung des Kündigungsschutzes gerechtfertigt wird.

Die prinzipielle Verbundenheit des Betriebsrats mit dem Betrieb und die unterlegene Verhandlungsposition können sich in unterschiedlichen Verhaltensweisen äußern. In zahlreichen Forschungen wurden typische Interaktionsmuster von Betriebsräten festgestellt. Interessant ist im Zusammenhang mit der Namensliste der von Schmidt und Trinczek beschriebene Betriebsratstyp, der eine Form des Co-Managements betreibe, indem er seine Interessen aus der Betriebsperspektive heraus formuliert. Die Leitfrage solcher Betriebsräte laute nicht mehr „Was ist gut für die Belegschaft?", sondern „Was ist gut für den Betrieb - und damit für die Belegschaft?" Dass diese Interessen nicht deckungsgleich sind, werde von ihnen nicht systematisch, sondern nur situativ reflektiert (Schmidt/ Trinczek 1999, S. 117). Eine überwiegend an den wirtschaftlichen Interessen des Betriebs orientierte Auswahl der Arbeitnehmer bei Kündigungen kann aber anders ausfallen als eine an den gesetzlichen Kriterien orientierte, in der sowohl wirtschaftliche als auch soziale Gesichtspunkte berücksichtigt sind (vgl. \$ 1 Abs. 2, 3 KSchG). Eine Besonderheit der Namensliste ist zudem, dass sie im Rahmen von Verhandlungen für einen Interessenausgleich abgeschlossen wird, eine Situation, in der der Betriebsrat besonders die betriebswirtschaftliche Situation „seines“ Betriebs im Auge haben muss. Verweigert er die Beteiligung an der Namensliste, gefährdet er möglicherweise den Abschluss des Interessenausgleichs.

Die häufig geringe Durchsetzungskraft von Betriebsräten, die auf die beschriebene Unterlegenheit zurückführbar sein dürfte, bestätigte auch eine neuere empirische Untersuchung von Nienhüser (2005). Bei einer Befragung von 1.000 Personalverantwortlichen wurden drei Fünftel der Betriebsräte von den Personalverantwortlichen als „schwach“ beschrieben, in dem Sinne, dass sie den Spielraum des BetrVG nicht ausnutzten. Von den „schwachen“ Betriebsräten wurden zugleich zwei Drittel als kooperativ wahrgenommen. Von den zwei Fünfteln der „starken“ Betriebsräte war etwa die Hälfte kooperativ. Ein Einfluss, der zu einvernehmlichen Vereinbarungen anhält, kann auch in dem Ideal von „winwin“-Lösungen gesehen werden, das sich für Konfliktsituationen zwischen Arbeitgeber und Betriebsrat durchgesetzt hat und es prinzipiell als nachteilig erscheinen lässt, auf bestehende Rechte zu pochen und einen Konflikt zu riskieren (Kremer 2008).

Das Problem, dass seine unterlegene Position es dem Betriebsrat im Fall von Namenslisten möglicherweise nicht gestattet, die Rechtmäßigkeit der Kündigungen sicherzustellen, wird auch in der rechtspolitischen Diskussion zum Teil anerkannt. Einige hegen die Hoffnung, dass Einigungsstellen, sofern sie an den Verhandlungen für den Interessenausgleich beteiligt sind, auf rechtliche Bedenken bei der Aufstellung einer Namensliste hinweisen. So sollen sie ein „Kungeln“ der Betriebspartner, bei dem Rechte einzelner Arbeitnehmer verletzt werden, verhindern (Kiel 2007).

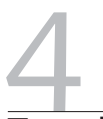

\section{Empirie}

Um Licht in die Blackbox „Namensliste“ $\mathrm{zu}$ bringen, hat das WSI Betriebsräte dazu interviewt. Im Rahmen der WSI-Betriebsrätebefragung 2007 machten aus einer Stichprobe von rund 45.000 Unternehmen 3.810 Betriebsräte in Betrieben mit 20 und mehr Beschäftigten Angaben dazu, ob es bei ihnen Namenslisten gab; letztlich wurden 181 Interviews mit Betriebsräten realisiert, in deren Betrieben in den letzten zehn Jahren eine oder mehrere Namenslisten im Sinne von $\$ 1$ Abs. 5 KSchG aufgestellt wurden. Betriebsräte, in deren Betrieben in diesem Zeitraum mehrere Namenslisten vorkamen, gaben Auskunft nur über die letzte dieser Namenslisten.

\subsection{ERFOLGSAUSSICHTEN VON KLAGEN}

Um überhaupt die Tragweite der umstrittenen Namenslisten einschätzen zu können, galt das Interesse der Untersuchung zunächst der Frage, wie sie sich auf die Erfolgsaussichten von Kündigungsschutzkla- gen der Arbeitnehmer auswirken. Laut der Befragung endeten $21 \%$ der Kündigungsschutzklagen von Arbeitnehmern, die auf einer Namensliste standen, mit einem abweisenden Gerichtsurteil. Allgemein enden $5 \%$ der Kündigungsschutzklagen mit abweisenden Urteilen (Höland et al. 2007, S. 142f.). Offen bleiben muss an dieser Stelle, ob die vergleichsweise hohe Zahl von Klageabweisungen tatsächlich überwiegend auf die vom Bundesarbeitsgericht angenommene häufigere Rechtmäßigkeit von Namenslistenkündigungen bei Betriebsratsbeteiligung zurückzuführen ist oder auf die Einschränkung der gerichtlichen Kontrolle. Die im Folgenden dargestellten Antworten auf die Frage nach der Möglichkeit der Einflussnahme der Betriebsräte auf die Auswahl der zu Kündigenden, nach den Motiven für eine Beteiligung an der $\mathrm{Na}-$ mensliste und auf weitere Fragen geben jedoch Anhaltspunkte dafür, dass die Erklärung für den hohen Anteil abweisender Urteile wohl in erster Linie in der Einschränkung der gerichtlichen Überprüfbarkeit zu sehen ist.

$5 \%$ der Kläger, die auf einer Namensliste standen, kehrten nach einer Klage aufgrund eines Urteils, eines gerichtlichen oder außergerichtlichen Vergleichs auf ihren Arbeitsplatz zurück. Die Rückkehrerquote allgemein liegt bei rund $15 \%$ (ebd., S. 204). Höher als bei Kündigungsschutzklagen allgemein war bei den Klageverläufen mit Namenslisten der Anteil der Arbeitnehmer, deren Klage mit dem Erhalt einer Abfindung endete. Auf $74 \%$ der Arbeitnehmer traf dies zu, während der entsprechende Anteil allgemein bei Kündigungsschutzklagen bei $50 \%$ liegt (ebd., S. 183). Der hohe Abfindungsanteil bei Namenslisten ist auch darauf zurückzuführen, dass bei Betriebsänderungen in aller Regel ein Sozialplan aufgestellt wird, der Abfindungen vorsieht. Insgesamt zeigt sich an dem deutlich höheren Anteil abweisender Urteile und der ebenso deutlich niedrigeren Rückkehrerquote, dass die Erfolgsaussichten einer Kündigungsschutzklage durch eine Namensliste verschlechtert werden.

Dieser Befund wird auch dadurch bestätigt, dass schon von vornherein die Zahl der Arbeitnehmer, die bei Vorliegen einer Namensliste gegen ihre Kündigung klagen, nur halb so groß ist wie die allgemeine Klagequote. Die Betriebsrätebefragung zur Namensliste ergab eine Klagequote von $7 \%$, während die allgemeine Klagequote 
gegen Arbeitgeberkündigungen bei rund $15 \%$ liegt (Pfarr et al. 2005, S. 58). Arbeitnehmer schätzen demnach - gegebenenfalls mithilfe sachkundiger Beratung - ihre Erfolgsaussichten bei einer Namensliste von vornherein geringer ein und nehmen eher von einer Klage Abstand.

\subsection{MOTIVE UND HANDLUNGS- MÖGLICHKEITEN DER BETRIEBSRÄTE}

Die Handlungsmacht eines Betriebsrats gegenüber dem Arbeitgeber zeigt sich unter anderem daran, ob er sich Anliegen des Arbeitgebers widersetzen bzw. ob er bei Verhandlungen mit dem Arbeitgeber seine eigenen Anliegen umsetzen kann. Diesen Aspekten galten die im Folgenden ausgewerteten Fragen. Auch die Frage, was Betriebsräte motiviert, sich an Namenslisten zu beteiligen, gehört in diesen Zusammenhang.

\section{INITIATIVE}

Von wem die Initiative für eine Namensliste ausging, ist eines der Indizien dafür, welche Seite vor allem ein Interesse am Abschluss der Namensliste hatte. Die Befragung ergab, dass die Initiative zum Abschluss in $45 \%$ der Fälle vom Arbeitgeber ausging, in $6 \%$ der Fälle vom Betriebsrat. $48 \%$ kamen auf Initiative von beiden Seiten zustande. Die Zahlen bestätigten die Vermutung, dass die Namensliste häufiger ein Anliegen des Arbeitgebers ist.

\section{WEIGERUNG}

Auch die Frage, ob es schon einmal in Fällen betriebsbedingter Mehrfachentlassungen vorkam, dass der Arbeitgeber eine $\mathrm{Na}$ mensliste wünschte, diese aber nicht $\mathrm{zu}$ stande kam, weil der Betriebsrat sich geweigert hat, zielte auf die Feststellung, wie groß tatsächlich die Möglichkeit ist, die Beteiligung an einer Namensliste abzulehnen. Sie wurde von $16 \%$ der Betriebsräte mit $J a$ beantwortet, $79 \%$ antworteten Nein. ${ }^{2}$ Die Angabe „Nein“ ist allerdings insofern nur eingeschränkt aussagekräftig, als sie eine unbekannte Anzahl von Fällen umfasst, in denen der Arbeitgeber nie eine Namensliste gewünscht hatte. Die Frage nach den Gründen für die Ablehnung zeigt, dass für einen Teil der Betriebsräte tatsächlich die Rechtmäßigkeit der Kündigungen so entscheidend war, dass gerade an diesem Punkt die Beteiligung scheiterte: $20 \%$ sagten sinngemäß, dass sie die Sozialauswahl als rechtswidrig beurteilt hatten, und weitere $6 \%$ sagten sinngemäß, dass sie die betriebliche Notwendigkeit der Kündigungen nicht gesehen hätten. Diese Betriebsräte haben sich so verhalten wie von der Rechtsprechung angenommen, denn bei Namenslisten eine Richtigkeitsgewähr bzgl. der Kündigungen anzunehmen, geht zwangsläufig einher mit der Erwartung, dass ein Betriebsrat rechtswidrige Kündigungen nicht mitträgt. $44 \%$ der Betriebsräte, die eine Beteiligung abgelehnt hatten, sagten sinngemäß, dass sie die Beteiligung aus Prinzip abgelehnt hätten, nämlich wegen der drohenden Einschränkung des Kündigungsschutzes oder weil sie nicht die Verantwortung für Kündigungen mit übernehmen wollten, sich nicht den „Schwarzen Peter" für die Kündigungen zuschieben lassen wollten, weil Kündigungen Aufgabe des Arbeitgebers seien oder weil sie schlicht keine Veranlassung zu einer Beteiligung an der Namensliste gesehen hätten. ${ }^{3}$ Diese Antworten zeugen von einer Auseinandersetzung mit den nachteiligen Wirkungen der Namenslisten und zugleich von der Weigerung, die Kündigungen mitzutragen, unabhängig davon, ob diese sich als rechtmäßig darstellen oder nicht. Sie offenbaren eine ablehnende Haltung gegenüber dem vom Gesetzgeber mit der Regelung verfolgten Anliegen.

\section{KENNTNIS UND EINFLUSS}

Bei Betriebsräten, die Namenslisten abschlossen, war problematisch, dass $15 \%$ von ihnen nicht bekannt war, dass durch die Namensliste der Kündigungsschutz beschränkt wurde ( $82 \%$ bejahten diese Frage). Hier offenbart sich, dass die Namenslisten auch von Betriebsräten getragen werden, die sich ihrer Wirkungsweise nicht bewusst waren. $7 \%$ der Betriebsräte konnten keinen Einfluss auf die Auswahl der Arbeitnehmer nehmen, die auf die Namensliste gesetzt wurden ( $91 \%$ bejahten die Möglichkeit zur Einflussnahme). Auch diese Betriebsräte hatten offensichtlich keine Möglichkeit, die Rechtmäßigkeit der Kündigungen sicherzustellen. Insgesamt waren es $20 \%$ der Betriebsräte, die entweder keinen Einfluss auf die Auswahl nehmen konnten oder denen nicht bewusst war, dass sie damit die Kündigungsschutzmöglichkeiten der Arbeitnehmer einschränken. Dieser Anteil muss zu Zweifeln an der Rechtmäßigkeitsgewähr von Namenslisten veranlassen, denn er zeigt, dass die tatsäch- liche Praxis nicht immer den Vorstellungen der Rechtsprechung entspricht. Die Annahme einer Richtigkeitsgewähr würde voraussetzen, dass ein Betriebsrat die Möglichkeit hat, vorgesehene Kündigungen, die rechtlich zweifelhaft sind, durch andere austauschen zu lassen, und dass er sich der rechtlichen Wirkung der Namensliste bewusst ist.

Für $12 \%$ der Betriebsräte war es auch ein Grund der Namensliste zuzustimmen, dass sie das Verhältnis zum Arbeitgeber nicht belasten wollten (von $87 \%$ wurde diese Frage verneint). Auch in diesen Fällen, in denen der Betriebsfrieden im Vordergrund stand, könnte die Rechtmäßigkeitskontrolle zurückgetreten sein.

\section{GEGENLEISTUNGEN}

Um Auskunft über die Motive der Betriebsräte zu erhalten, wurde danach gefragt, $\mathrm{ob}$ sie im Austausch gegen eine finanzielle Aufstockung der Sozialplanabfindungen, gegen die Gründung einer Beschäftigungsgesellschaft oder gegen Beschäftigungssicherungen für verbleibende Beschäftigte der $\mathrm{Na}$ mensliste zugestimmt hatten. Natürlich konnten auch mehrere dieser Alternativen zutreffen. Anschließend wurde gefragt, welche sonstigen Gründe die Betriebsräte zum Abschluss der Namensliste veranlasst hatten. Es zeigte sich, dass der Arbeitgeber in $62 \%$ der Namenslisten als Gegenleistung die Sozialplanabfindungen erhöht (in 34 \% nicht), in $19 \%$ als Gegenleistung eine Beschäftigungsgesellschaft eingerichtet (in $79 \%$ nicht) und in $64 \%$ Beschäftigungssicherungen für die verbleibende Belegschaft zugestimmt hat (in $34 \%$ nicht). Bei $82 \%$ der Namenslisten hatte der Arbeitgeber eine oder mehrere der genannten Gegenleistungen angeboten. Wie oben beschrieben, lösen solche Gegenleistungen Situationen aus, in denen der Betriebsrat nur schwer das Interesse möglicherweise unrechtmäßig Gekündigter am Erhalt des vollen Kündigungsschutzes den Belangen der Mehrheit voranstellen kann. Die Befragung belegt die starke Verbreitung solcher Gegenleistungen

2 Diese Prozentangabe bezieht sich auf alle 1.419 der befragten Betriebe, die in den letzten zehn Jahren ein- oder mehrmals Mehrfachentlassungen vorgenommen hatten

3 Bei den Antworten zu den „offenen“ Fragen umfassen die zu den vollen $100 \%$ fehlenden Anteile die Antworten, die zu keiner der hier gebildeten Kategorien passten. 
des Arbeitgebers, die es fraglich erscheinen lassen, dass der Betriebsrat seine Zustimmung zur Namensliste an die Bedingung knüpfen konnte, dass alle Kündigungen rechtmäßig erfolgten: Insgesamt mehr als vier Fünftel der Namenslisten hatte der Arbeitgeber sich durch eine Gegenleistung erkauft.

\section{SONSTIGE BEWEGGRÜNDE}

Neben solchen Tauschgeschäften gab es weitere Motive, die den Betriebsrat zum Abschluss der Namensliste motivierten. $24 \%$ der Betriebsräte, die weitere Motive nannten, gaben als sonstiges Motiv sinngemäß an, dass sie Arbeitsplätze sichern und deshalb den Bestand des Betriebs gewährleisten oder sogar eine drohende Betriebsschließung verhindern wollten; $9 \%$ sagten, dass sie zu einer größeren Leistungsfähigkeit des Betriebs beitragen wollten. Die Antworten dieses Drittels jener Betriebsräte mit weiteren Motiven bestätigen die beschriebene starke Verbundenheit zum Betrieb, die im Übrigen auch schon eine Rolle spielt, wenn Beschäftigungssicherungen als Angebot des Arbeitgebers im Raum stehen. Die Antworten deuten auf eine Art der Interessenwahrnehmung hin wie sie von Schmidt/Trinczek als Co-Management beschrieben wurde, bei der der Betriebsrat danach fragt, was gut für den Betrieb und deswegen auch gut für die Belegschaft ist. Die Verfolgung betriebswirtschaftlicher Interessen durch Entlassungen ist aber auch ohne Namenslisten möglich; die Voraussetzungen für betriebsbedingte Kündigungen sind in $\$ 1$ Abs. 2, $3 \mathrm{KSchG}$ abschließend enthalten, der keine Namensliste voraussetzt. Wenn ein Betriebsrat wie hier auf das Wohl des Betriebs abstellt, hat er sich allerdings womöglich nur mit der Namensliste einverstanden erklärt, weil er die Kündigungen auch für rechtmäßig hielt. Jedoch zeigen Daten zu Kündigungsschutzverfahren allgemein (mit und ohne Namensliste), dass von jenen betriebsbedingten Kündigungen, die ohne Widerspruch des (vorhandenen) Betriebsrats zustande gekommen und durch Urteil entschieden worden sind, immerhin $53 \%$ per Urteil für unwirksam erklärt wurden. ${ }^{4}$ Auch die Beurteilung einer Kündigung als rechtmäßig durch einen Betriebsrat garantiert demnach nicht deren Rechtmäßigkeit bei gerichtlicher Überprüfung. Dass einem Gericht ganz andere Mittel zur Rechtmäßigkeitsbeurteilung zur Verfügung ste- hen als einem Betriebsrat, ist eigentlich selbstverständlich.

$12 \%$ der Betriebsräte nannten als sonstiges Motiv, die Einhaltung der Sozialauswahl sichern $\mathrm{zu}$ wollen. Dieses Motiv kommt der Idee nahe, dass die Namenslistenregelung eine Richtigkeitsgewähr bietet. Der Arbeitgeber ist aber auch sonst zur Sozialauswahl gesetzlich verpflichtet. Es ist zwar sinnvoll, dass der Betriebsrat sich schon vor Ausspruch der Kündigung um eine fehlerfreie Sozialauswahl bemüht. Jedoch gibt $\$ 102$ Abs. 3 Nr. 1 BetrVG dem Betriebsrat auch ohne Namensliste bei fehlerhafter Sozialauswahl das Recht zum Widerspruch gegen eine Kündigung.

$6 \%$ der Betriebsräte nannten als sonstiges Motiv, dass so die Zahl der ursprünglich für eine Entlassung vorgesehenen Arbeitnehmer reduziert werden konnte; $3 \%$ sagten, dass sie den Arbeitgeber zum Eintritt in den Arbeitgeberverband oder zur Gewährung zusätzlicher Tarifleistungen bewegen wollten. Diese beiden Motive ähneln dem Erhalt einer Gegenleistung wie z. B. einer Beschäftigungssicherung.

$1 \%$ der Betriebsräte nannten schließlich als sonstiges Motiv, dass sie nach vorheriger Abstimmung mit den Arbeitnehmern nur diejenigen auf die Namensliste setzen ließen, die ohnehin aufhören wollten. Hier wird die Namensliste als eine "günstige Gelegenheit" genutzt und als Möglichkeit, in einer Situation anstehender Kündigungen das geringste Übel zu wählen. Hierzu müssen aber die tatsächlichen Voraussetzungen gegeben sein (genügend Arbeitnehmer, die ohnehin aufhören wollen), was vom Zufall abhängt, wie auch der geringe Anteil dieser Antwort bestätigt.

\subsection{KORREKTIV EINIGUNGSSTELLE?}

Die teilweise geäußerte Hoffnung trügt, dass Einigungsstellen in den Verhandlungen um Namenslisten auf rechtliche Bedenken hinweisen und ein „Kungeln“ der Betriebspartner, bei dem Rechte einzelner Arbeitnehmer verletzt werden, verhindern können. Die Befragung ergab, dass nur in $8 \%$ der Fälle, in denen eine Namensliste aufgestellt wurde, eine Einigungsstelle an den Verhandlungen zum Interessenausgleich beteiligt war (in $91 \%$ der Fälle nicht). Damit ist auch die Möglichkeit zu einer korrigierenden Einwirkung der Einigungsstelle nur in den wenigsten Fällen gegeben.

\subsection{REAKTIONEN DER BELEGSCHAFT}

Die Befragung bestätigte schließlich auch die Vermutung, dass die verbleibende Belegschaft die Beteiligung an einer Namensliste überwiegend nicht missbilligt. $15 \%$ der Betriebsräte bejahten die Frage, ob die verbleibende Belegschaft negativ auf die Namensliste reagiert habe. Dagegen habe es in $24 \%$ der Fälle sogar positive Reaktionen gegeben, in den meisten Fällen ( $55 \%$ ) hielten sich laut Betriebsrat die positiven und negativen Reaktionen die Waage. Der relativ geringe Anteil negativer Reaktionen lässt sich wohl mit der Erleichterung der Verbleibenden erklären, selbst von einer Kündigung verschont geblieben zu sein.

\section{Schlussfolgerungen}

Mit Blick auf die Rechte, die das Betriebsverfassungsgesetz dem Betriebsrat eröffnet, auf die Funktionen, mit denen es ihn betraut, und auf seine tatsächliche Position im Betrieb stellt die Namenslistenregelung für den Betriebsrat ein schwer aufzulösendes Dilemma dar. Er kann gezwungen sein, sich zwischen Rechtskontrolle und betriebswirtschaftlichen Interessen zu entscheiden. Da der Betriebsrat die ihm durch die Regelung übertragene Verantwortung strukturell bedingt häufig nicht wahrnehmen kann, sollte noch einmal über $\S 1$ Abs. 5 KSchG nachgedacht werden. Zwar kommt es Arbeitgebern entgegen, dass bei Existenz von Namenslisten noch weniger Arbeitnehmer gegen ihre Kündigung klagen und mehr Klagen erfolglos sind. Die von der Einschränkung des Kündigungsschutzes erwartete positive Beschäftigungswirkung der Regelung wird jedoch nicht erreicht. Die Regelung sollte daher aufgehoben werden. Alternativ denkbar wäre allenfalls, ähnlich der Regelung beim Interessenausgleich, die zwingende Beteiligung einer Einigungsstelle gesetzlich vorzusehen. Auf diese Weise würde eine unabhängige und rechtsberatende Stelle den Prozess um den Abschluss einer Namensliste begleiten können. Keinesfalls sollte dabei jedoch die Namensliste rechtlich erzwingbar gestaltet werden.

\footnotetext{
4 Eigene Berechnungen anhand des Datensatzes des
} Forschungsprojekts Küprax (Höland et al. 2007). 


\section{LITERATUR}

Bundesarbeitsgericht (BAG) (1987): Urteil vom 20.11.1987, 2 AZR 284/86

Bundesarbeitsgericht (BAG) (1996): Urteil vom 06.11.1996, 5 AZR

334/95

Bundesarbeitsgericht (BAG) (2007): Urteil vom 19.6.2007, 2 AZR

304/06

Deutscher Bundestag (1996): Entwurf eines arbeitsrechtlichen Gesetzes zur Förderung von Wachstum und Beschäftigung, BT-Drucksache $13 / 4612$

Deutscher Bundestag (2003): Entwurf eines Gesetzes zu Reformen am Arbeitsmarkt, BT-Drucksache 15/1204

Gamillscheg, F. (2008): Kollektives Arbeitsrecht. Band II: Betriebsverfassung, München

Henssler, M./Preis, U. (2007): Entwurf eines Arbeitsvertragsgesetzes,

Neue Zeitschrift für Arbeitsrecht, Beilage 1

Höland, A. (2002): Gesetzesrecht, Tarifrecht, Betriebsrecht, in: Seifert, H. (Hrsg.): Betriebliche Bündnisse für Arbeit, Berlin, S. 39-63

Höland, A./Kahl, U./Zeibig, N. (2007): Kündigungspraxis und Kündigungsschutz im Arbeitsverhältnis, Baden-Baden

Kappenhagen M. (2004): Die neue "alte" Namensliste nach § 1 Abs. 5 KSchG, Fachanwalt Arbeitsrecht 2, S. 37-39
Kiel, H. (2007): Namenslisten und Beendigungskündigung, Presseinformation zu einem Vortrag (unveröffentlicht)

Kittner, M./Däubler, W./Zwanziger, B. (2004): Kündigungsschutzrecht, $\mathrm{KSchR}, 6$. Auflage, Frankfurt/M.

Kremer, S. (2008): Bewusstseins- und Verhaltensänderungen durch die "Flexibilisierung" des Arbeitsrechts, Baden-Baden

Nienhüser, W. (2005): Der Einfluss des Betriebsrats-Typs auf die Nutzung und Bewertung von Betriebsvereinbarungen, in: Industrielle Beziehungen 1, S. 5-27

OECD (1999): Employment Outlook, Paris

OECD (2006): Employment Outlook, Paris

Pfarr, H./Ullmann, K./Bradtke, M./Schneider, J./Kimmich, M./Bothfeld, S. (2005): Der Kündigungsschutz zwischen Wahrnehmung und Wirklichkeit, München und Mering

Reufels, M. (2007): Prozesstaktik im Arbeitsrecht, Baden-Baden Schmidt, R./Trinczek, R. (1999): Der Betriebsrat als Akteur der industriellen Beziehungen, in: Müller-Jentsch, W. (Hrsg.): Konfliktpartnerschaft, München und Mering, S. 103-128

Thüsing, G./Wege, D. (2005): Freiwilliger Interessenausgleich und Sozialauswahl, Betriebs-Berater 4, S. 213-216 